\title{
Retinopathy of prematurity and neonatal gut microbiome: An interview with Professor Dimitra Skondra, Associate Professor of Ophthalmology and Vitreoretinal Surgeon at The University of Chicago (USA)
}

\author{
IOANNIS N. MAMMAS ${ }^{1-4}$ and DEMETRIOS A. SPANDIDOS ${ }^{1,3}$ \\ ${ }^{1}$ Institute of Paediatric Virology; ${ }^{2}$ Paediatric Clinic, Aliveri, 34500 Island of Euboea; \\ ${ }^{3}$ Laboratory of Clinical Virology, Medical School, University of Crete, 71003 Heraklion; \\ ${ }^{4}$ First Department of Paediatrics, University of Athens School of Medicine, 11527 Athens, Greece
}

Received July 31, 2020; Accepted October 24, 2020

DOI: $10.3892 /$ etm.2020.9424

\author{
'Oh light of my eyes' \\ $[\hat{\Omega} \phi \hat{\omega} \varsigma \tau \hat{\omega} v \dot{o} \phi \theta \alpha \lambda \mu \hat{\omega} v \mu o v)$ \\ Epitaphios Hymn
}

\begin{abstract}
Retinopathy of prematurity (ROP) is a sightthreatening disorder of the retina affecting neonates of very low birth weight and gestational age, and is characterized by the development of abnormal blood vessel growth. According to Dr Dimitra Skondra, Associate Professor of Ophthalmology and Vitreoretinal Surgeon at the University of Chicago School of Medicine in Chicago, USA, the neonatal gut microbiome may be implicated in the neoangiogenesis process in the neonatal retina and this role may be one of the missing links in the pathogenesis of ROP. The human gut microbiome consists of bacteria, viruses, protozoa and fungi, which colonize the sterile fetal intestine, and differ depending on gestational age, mode of delivery, type of neonatal feeding, the usage of antibiotics and the requirement of neonatal intensive care. To date, it has been related to multiple nutritive, metabolic and immunological functions and has been implicated in the pathogenesis of several human diseases, such as the inflammatory bowel diseases, autoimmune and neurogenerative disorders, metabolic syndrome, cardiovascular diseases and various types of malignant neoplasias. Recent research has proposed that the neonatal gut microbiome profile in high-risk neonates who develop ROP is significantly enriched with
\end{abstract}

Correspondence to: Professor Demetrios A. Spandidos, Laboratory of Clinical Virology, Medical School, University of Crete, 71003 Heraklion, Greece

E-mail: spandidos@spandidos.gr

Key words: ROP, retinopathy of prematurity, neonatal microbiome, probiotics, personalized medicine
Enterobacteriacaea species several weeks prior to the diagnosis of ROP. Further research using animal models is required to prove the causative or secondary role of the microbiome composition in the development and clinical course of ROP. If this role is proven, the gut microbiome could then be a target of intervention for personalized medicine in the prevention and therapeutic management of ROP in neonates.

\section{Contents}

1. Introduction

2. Questions and Answers

\section{Introduction}

Retinopathy of prematurity (ROP) is a sight-threatening disorder of the developing blood vessels in the retina of neonates of very low birth weight and gestational age $(1,2)$. It is characterized by an initial phase of vasoconstriction and obliteration of the capillary retinal network beginning after birth, which later on progress to vasoproliferation causing retinal tension, distortion and detachment (3). To date, the role of neoangiogenesis pathways in the human retina involving the vascular endothelial growth factor (VEGF) and insulin-like growth factor-1 (IGF-1) pathways has been excessively studied (1). ROP has been proposed to be multifactorial; the principal risk factors for its development are birth weight and gestational age. Treatment strategies against ROP include the usage of laser photocoagulation and cryotherapy, while recently the intravitreal usage of anti-VEGF has also been introduced into clinical practice for ROP patients $(4,5)$. Despite current screening, advanced neonatal care and improved management 
guidelines, ROP remains a leading cause of childhood blindness and visual disability, worldwide $(1,2,6)$.

Dr Dimitra Skondra, MD, PhD (Fig. 1), Associate Professor of Ophthalmology at the University of Chicago School of Medicine in Chicago (USA), is a highly respected, board-certified Retina Specialist, with a particular focus on the medical and surgical treatment of vitreoretinal diseases. She studied medicine at the University of Crete School of Medicine, where she received her MD and her PhD (7) and she specialized in Ophthalmology at Harvard Medical School, Massachusetts Eye and Ear Infirmary, Boston, MA and Weill Cornell Medical College, New York Presbyterian Hospital, New York City, NY in the USA. She is an expert in delivering care for diabetic eye disease, retinal detachment, age-related macular degeneration, retinal vein occlusions, eye trauma, proliferative vitreoretinopathy and intraocular infections, with advanced training and expertise in surgical repair of complex diabetic retinal detachments. In addition to her clinical expertise, Dr Skondra is an active researcher. As a physician-scientist, she is dedicated to investigating methods to improve treatment strategies for retinal conditions. She is currently engaged in clinical and translational research in diabetes and other retinal diseases with special focus on the role of the gut microbiome in retinal diseases. Her research in retinal imaging aims to provide a better understanding of the pathogenesis, early detection and identification of prognostic information for to enable more targeted intervention and treatment. She has performed 36 publications in peer-reviewed journals (8-43), has given numerous invited talks in national and international meetings and serves as scientific reviewer for several ophthalmology journals. Throughout her career, she has received several prestigious awards, including the 2019 The Association for Research in Vision and Ophthalmology (ARVO) Genentech Age-related Macular Degeneration (AMD) Translational Award, the University of Chicago Ophthalmology and Visual Science (OVS) Innovation Award, the Retina Society Raymond Margherio Award, the Alcon Harvard Medical Scholl Ophthalmology Clinical Research Scholar Award, the Association of University Professors of Ophthalmology (AUPO) Research Award, the ARVO/Alcon Early Career Clinician Scientist Award, the Joslin-Tonseth Research Fellowship Award and the Knights Templar Award. She is an elected member of the prestigious Macula Society (https://www.maculasociety.org), the Retina Society (https://www.retinasociety.org) and is an active member of the American Society of Retinal Specialists, the American Academy of Ophthalmology, the Vitreous Buckle Society and the Association of Research in Vision and Ophthalmology.

Dr Skondra is the team leader of the Retina Microbiome Team (RMT) of the University of Chicago School of Medicine, focusing on human ocular and extraocular microbiome and its role in the pathogenesis and treatment of retinal diseases; these include AMD and ROP (18). Moreover, her research aims to clarify the potential interaction of the human microbiome with the angiogenesis pathways in the retina and to identify the protective or pathogenic role of different bacterial species in the development of different vascular retinal disorders in adults and neonates, including ROP. On Saturday, September 14th, 2019, she participated at 'The Retina Society 2019 Annual Meeting' in London, UK, where she presented her work on the

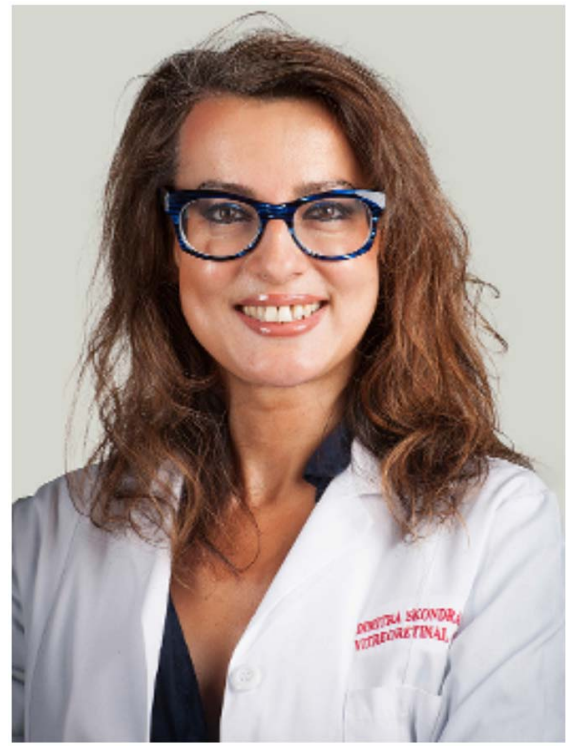

Figure 1. Professor Dimitra Skondra, Associate Professor of Ophthalmology and Vitreoretinal Surgeon at The University of Chicago School of Medicine in Chicago, USA.

neonatal intestine microbiome and ROP (44). In the context of the forthcoming '6th workshop on paediatric virology' organized by the Institute of Paediatric Virology (IPV) Dr Skondra will give a plenary lecture on 'ROP and neonatal microbiome'. This article presents her teleconference meeting with the Paediatric Virology Study Group (PVSG) on neonatal microbiome organized by the IPV on Monday, April 13th, 2020.

\section{Questions and Answers}

Question: Dear Professor Dimitra Skondra, first of all I would like to congratulate you for your recent exemplary lecture in London last September on retinopathy of prematurity (ROP). Despite current improvements in perinatal and neonatal care, ROP remains a major cause of neonatal blindness, worldwide. What is ROP, how is it developed in premature neonates and what factors are implicated in its pathogenesis?

Answer: First of all, Ioannis, thank you for this invitation; it is always great to catch up and discuss with good colleagues and friends from Greece. Retinopathy of prematurity (ROP) is a neovascular process that is developing in high-risk neonates of very low birth weight and low gestational age. It is characterized by the presence of undeveloped retinal vessels at the initial phase and the development of abnormal blood vessels growth later on. Up to 20-30\% of high-risk infants with extremely low birth weight of less than $500 \mathrm{~g}$ or very low gestational age of less than 27 weeks develop ROP. Most babies who develop ROP do not meet the criteria for treatment and ROP regresses spontaneously without any blindness consequences. However, we still do not know why some high-risk infants develop severe ROP, while other high-risk infants do not - even they have similar known risk factors for ROP.

Question: In your lecture in London, you presented data on the relationship between ROP and the neonatal intestine microbiome. What is the human microbiome? What are the genera, 
species and strains of bacteria and other microorganisms, which compose it?

Answer: The human microbiome has been there for many many years, but in medicine we were used to ignoring it, until almost 10-20 years ago. There are trillions of microbes living in the human body, which represent almost one third of the total average human body mass. The human gut microbiome consists of bacteria, viruses, protozoa and fungi, which are present in the human gastrointestinal tract (GIT) and represent the vast majority of the human microbiome. Here, in Chicago, at the University of Chicago School of Medicine, we have established the RMT, looking into how the gut microbiome is implicated in retinal disorders and angiogenesis, which is the growth of abnormal retinal vessels in retina and choroid, hallmark sign of blinding retinal conditions like ROP and AMD and diabetic retinopathy. Our principal question of our research project, which was presented in London on September, was whether the human gut microbiome would potentially associated with the development or the risk for development of ROP.

Question: When does the colonization of the intestine microbiome occur in neonates and how is it altered during the first period of life?

Answer: The colonization of the gut microbiome in the sterile foetal intestine occurs during and after the birthing process. There are some researchers, who have proposed that microbial colonization occurs in utero, but this is debatable. Gestational age is considered as the most significant factor for the development of gut microbiome. Other factors that have an impact on the proliferation of microbes include the mode of delivery, usage of antibiotics, admission in the neonatal intensive care unit (NICU), type of infant feeding (breast, formula or parenteric). For example, the neonatal microbiome differs significantly in neonates born with Caesarian section compared to neonates delivered vaginally. The admission of preterm neonates at the NICU is also characterized by prolonged antibiotic therapy, parenteral nutrition, delayed oral feeding and intubation, affecting the composition of neonatal gut microbiome. Antibiotics influence the neonatal microbiome, delaying and altering its normal intestinal colonization.

Question: The intestinal microbiome has been implicated in several nutritive, metabolic and immunological functions. According to the literature, in what diseases has the intestine microbiome been found to be involved?

Answer: Over the past decade, more and more studies have investigated the crucial role of the human gut microbiome in the mechanism of health, as well as in the pathogenesis of several diseases. The initial studies focused on the relationship between the gut microbiome and gastrointestinal diseases, such as the inflammatory bowel disease - Crohn's disease and ulcerative colitis. It also has an important role in metabolic syndrome, obesity, as well as in neurodegenerative diseases, such as Parkinson's disease, Alzheimer's disease, cardiovascular diseases, multiple sclerosis, rheumatoid arthritis, asthma, food allergies and cancer. Moreover, the gut microbiome plays an important role in nutrient and mineral absorption, synthesis of enzymes, vitamins and amino acids, circadic rhythm, brain development, weight gain, bone growth and of course immunity.
Question: What is the role of the human intestine microbiome in immunity?

Answer: The human gut microbiome enhances epithelial barrier integrity and provides immunomodulation and protection against multiple pathogens. In patients with inflammatory bowel disease or autoimmune disorders or transplant rejection this relationship is more than crucial. The human gut microbiome controls human immune system reaction and its immunological role is significant in several diseases, even in unexpected malignant neoplasias, such as melanoma. For example, different characteristics of the gut microbiome have been reported in patients with metastatic melanoma who respond to immunotherapy compared to those who do not respond.

Question: Which is the principal mechanism of the establishment of this very important multi-systemic role of the gut microbiome?

Answer: Basically, though different metabolites, such as short-chain fatty acids (SCFAs), that different microbes secrete into the GIT. These metabolites are implicated in several biochemical pathways involved in multiple nutritive, metabolic and immunological functions.

Question: So, is neonatal microbiome related to ROP? What were the results of your research?

Answer: The initial idea of our research was to examine if the neonatal gut microbiome is one of the missing links in the pathogenesis of ROP. This was really a mystery question trying to answer why some high-risk neonates develop ROP, while others do not. We established collaborations with Dr Erika Claud, Professor of Pediatrics and Director of Neonatology Reasearch and Dr Sarah Rodriguez, Assistant Professor of Opthalmology and Visual Science and co-director of ROP service and our RMT, where I have the role of team leader. We decided to focus on very low weigh birth babies of less than $750 \mathrm{~g}$ and very low gestational age of less than 27 weeks and evaluated their neonatal intestine microbiome from the date of birth until the age, when the diagnosis of ROP is usually performed, around 36 weeks. We selected these neonates and weekly samples were collected. We analyzed our data comparing high-risk infants who developed severe ROP and received treatment to those high-risk infants who did not develop any ROP. Looking at these very extreme groups, we interestingly found statistically significant differences in their microbiome profile at the age of 28 weeks. The microbiome profile in infants who developed severe ROP was significantly enriched with Enterobacteriaceae species. The early microbiome profile seemed to be different, but not the profiles later on. Looking the sequencing data of these microbes, we also examined potential pathways that specific microbes and their genes may be involved in the pathogenesis of ROP and we found several metabolic pathways especially regarding amino acid synthesis to be enriched in the group without ROP.

Question: However, you did not prove if this is a causative relationship or simply a co-incidence.

Answer: You are right. Our findings were based on a pilot, observational study, which was clinical and provided only preliminary evidence. The fact that we saw differences does 
not prove, indeed, the causative role of the neonatal microbiome in ROP. However, our observation that the early microbiome profile seems to be different between babies who develop ROP and those who do not is something that definitely needs further research. It was really interesting that neonates who developed ROP tended to be on a longer duration of antibiotics. The theory that sepsis predisposes to ROP could be related to exposure with broad spectrum antibiotics, very early on. It disrupts the normal microbiome development, its diversity and the normal ration between protective and pathogenic microbes, which compose it. This gut microbiome disruption can be implicated in pathways that are related to new blood vessels growth and ROP development. On the other edge of the spectrum, studying the effects of human gut microbiome in angiogenesis of the retinal vascular disorders in older adults with various ophthalmic diseases such as AMD, we have already seen that the human gut microbiome can directly affect the gene expression of VEGF and IGF pathways in the retina, which are implicated in the angiogenesis process. However, we still have to answer if this is a causative or a secondary effect and how is this related.

Question: In your research did you examine the impact of breast feeding on the development of ROP? Could you summarize the current evidence of the impact of human breast milk in the neonatal microbiome and its protective effects on infants? Answer: Yes, we included it in our data collection, as well as in our multivariate statistical analysis. To date, we know that breast feeding is protective against ROP. Babies who are breast-fed are more protected against ROP compared to those who are parenterally fed or receive formula nutrition. The diet of babies is very significant, indeed, and as I mentioned earlier, it is a significant modifying factor of the neonatal gut microbiome. Another reason that breast feeding is protective could be that it promotes a healthier gut microbiome, which is thought to suppress the growth of potentially pathogenic bacteria. It is very interesting to examine, though, the exact mechanism through which breast feeding is related to ROP and the exact sequel of different factors, including the neonatal gut microbiome, in the pathogenesis of ROP; further studies are needed to study this relationship with ROP.

Question: Could the understanding of this sequel be used in the prevention of ROP?

Answer: Hypothetically yes. The neonatal gut microbiome profiling at the date of birth could provide valuable information on the neonatal needs for a healthier neonatal microbiome balance. For example, if pathogenic species that are proven to be related to ROP are detected, they could be modified accordingly and replaced with non-pathogenic or protective species in order to prevent the development of ROP, as well as other diseases, such necrotizing enterocolitis, which has been excessively studied regarding the role neonatal gut microbiome in its pathogenesis.

Question: Viruses are also included in the neonatal microbiome. Have you studied the presence of specific viruses in neonatal intestine microbiome? Are there recent studies on the involvement of viruses in neonatal microbiome?

Answer: We have only studied bacterial species. Viruses are also very important, although their role is less known. To date, there are a number of studies examining the role of viruses in the gut and respiratory microbiome in adults, as well as in children and neonates. But, as far as I know, there are no studies available on the relationship between viral microbiome and ROP. This is a really interesting question that could be examined in the future.

Question: What are the current screening and therapeutic management options for patients with ROP? What is your experience in the management of neonates with ROP in Chicago, USA?

Answer: The proper screening of ROP of premature neonates with a gestational age of less than 30 weeks is very significant even the criteria keep on changing. It is performed by paediatric ophthalmology specialists being comfortable in examining the retina of premature neonates and identifying neonates with ROP. Early diagnosis of ROP can allow effective intervention before the retina is detached. There is a shift of the paradigm regarding the management of ROP in the last 5 years. The initial usage of cryotherapy in the avascular retina of neonates with ROP a couple of decades ago was replaced with laser therapy destroying the peripheral areas of the retina, slowing or reversing the abnormal growth of blood vessels. Recently, the use of intravitreal anti-VEGF therapy has caused a great revolution in the management of severe ROP cases trying to avoid very excessive laser therapies and their side-effects and complications. At the University of Chicago, the first-line management of cases with severe ROP by our paediatric ophthalmology specialist team tends to frequently include anti-VEGF injections. This strategy requires close follow-up of the patients, which helps to identify cases requiring supplementary laser therapy. The crucial, though, message for the management of ROP is the necessity for its early detection and treatment before the retina starting detaching!

Question: Could the understanding of human microbiome be used in the development of new prevention and therapeutic strategies against ROP and how?

Answer: Absolutely; this is just the beginning. First, we need to understand and prove if changing the neonatal microbiome composition really affects the clinical course of ROP. Then, we have to identify if there are specific microbes that are missing or are enriched related to the disease development and to examine if their modification changes the severity of ROP. If these are proven using animal models, then there could be several ways of therapeutic interventions aiming to a 'healthier' neonatal gut microbiome. There are numerous ways to manipulate the human gut microbiome once we prove which direction is effective. These interventions could provide specific protective strains or targeted pre-biotics or probiotics or other targeted supplements/metabolites or antibodies against pathogenic metabolites or other medications that affect microbiome. There is a completely new therapeutic era in this field and in the future this can be the key for personalized medicine in ROP.

Question: What will be the next step of your research?

Answer: The next step, now, is to work on animal models to see how vessels growth is related to gut microbiome. In my laboratory, we work with germ-free mice; these mice are the gold-standard animal models for studies of the microbiome 
and will enable us to answer our questions. Germ-free mice are bred in isolators, which fully block exposure to microorganisms keeping them free of detectable bacteria and viruses. We have developed the first angiogenesis animal model with laser in these mice and using specific protocols we have found the way to develop new blood vessels in their retina and keep these mice sterile. We intend to examine how new blood vessels are grown with or without microbiome or with different modification of it. This animal model will help us to understand our unanswered questions regarding the role of the human gut microbiome in retinal angiogenesis and provide the proof of concept in our hypothesis.

Question: Thank you very much for this very interesting discussion. On behalf of the PVSG and our newly founded Institute of Paediatric Virology we would like to wish you all the best in your state-of-the-art clinical and research contribution in Ophthalmology! We look forward to your plenary lecture in our forthcoming '6th workshop on Paediatric Virology' focusing on 'ROP and neonatal microbiome'.

\section{Acknowledgements}

This article is published in the context of the foundation of the Institute of Paediatric Virology (IPV; https:// paediatricvirology.org) based on the island of Euboea (Greece), under the auspices of the World Academy of Sciences (WAS) and the support of the Department of Clinical Virology of the University of Crete School of Medicine and the First Department of Paediatrics of the University of Athens School of Medicine. We would like to thank all the members of the IPV for their valuable comments and corrections.

\section{Funding}

No funding was received.

\section{Availability of data and materials}

Not applicable.

\section{Authors' contributions}

INM and DAS contributed equally to the conception and design of this manuscript, wrote the original draft, edited and critically revised the manuscript, read and approved the final manuscript.

\section{Ethics approval and consent to participate}

Not applicable.

\section{Patient consent for publication}

Not applicable.

\section{Competing interests}

INM and DAS are co-founders of the Institute of Paediatric Virology (IPV). DAS is the Editor-in-Chief for the journal, but had no personal involvement in the reviewing process, or any influence in terms of adjudicating on the final decision, for this article.

\section{References}

1. Chen J and Smith LE: Retinopathy of prematurity. Angiogenesis 10: 133-140, 2007.

2. Fierson WM; American Academy of Pediatrics Section on Ophthalmology, American Academy of Ophthalmology, American Association for Pediatric Ophthalmology and Strabismus and American Association of Certified Orthoptists: Screening examination of premature infants for retinopathy of prematurity. Pediatrics 142: e20183061, 2018.

3. Cayabyab R and Ramanathan R: Retinopathy of prematurity: therapeutic strategies based on pathophysiology. Neonatology 109: 369-376, 2016.

4. Sankar MJ, Sankar J and Chandra P: Anti-vascular endothelial growth factor (VEGF) drugs for treatment of retinopathy of prematurity. Cochrane Database Syst Rev 1: CD009734, 2018.

5. Rodriguez SH, Peyton C, Lewis K, Andrews B, Greenwald MJ, Schreiber MD, Msall ME and Blair MP: Neurodevelopmental outcomes comparing bevacizumab to laser for type 1 ROP. Ophthalmic Surg Lasers Imaging Retina 50: 337-343, 2019.

6. Wilson CM, Ells AL and Fielder AR: The challenge of screening for retinopathy of prematurity. Clin Perinatol 40: 241-259, 2013.

7. Skondra D: Molecular inflammatory factors in diabetic retinopathy: a role of azurocidin (unpublished $\mathrm{PhD}$ thesis). University of Crete School of Medicine, 2014.

8. Chun LY, Massamba N, Silas MR, Blair MP, Hariprasad SM and Skondra D: Use of optical coherence tomography angiography in the diagnosis of small retina lesions in Von Hippel-Lindau disease. Eye (Lond): February 10, 2020 (Epub ahead of print).

9. Chun LY, Dolle-Molle L, Bethel C, Dimitroyannis RC, Williams BL, Schechet SA, Hariprasad SM, Missiakas D, Schneewind O, Beavis KG, et al: Rapid pathogen identification and antimicrobial susceptibility testing in in vitro endophthalmitis with matrix assisted laser desorption-ionization Time-of-Flight Mass Spectrometry and VITEK 2 without prior culture. PLoS One 14: e0227071, 2019.

10. Massamba N, Sellam A, Butel N, Skondra D, Caillaux V and Bodaghi B: Use of fundus autofluorescence combined with optical coherence tomography for diagnose of geographic atrophy in age-related macular degeneration. Med Hypothesis Discov Innov Ophthalmol 8: 298-305, 2019.

11. Golas L, Skondra D, Ittiara S, Bajic N, Jeng-Miller KW, Mukai S, Yonekawa Y and Blair MP: Efficacy of retinal lesion screening in Von Hippel-Lindau patients with widefield color fundus imaging versus widefield FA. Ophthalmic Surg Lasers Imaging Retina 50: e260-e265, 2019.

12. Chun LY, Silas MR, Dimitroyannis RC, Ho K and Skondra D: Differences in macular capillary parameters between healthy black and white subjects with optical coherence tomography angiography (OCTA). PLoS One 14: e0223142, 2019.

13. Komati R, Schechet S and Skondra D: Multimodal imaging of dissociated optic nerve fiber layer after internal limiting membrane peel. Ophthalmol Retina 3: 776, 2019.

14. Sokol JT, Schechet SA, Rosen DT, Ferenchak K, Dawood S and Skondra D: Outcomes of vitrectomy for diabetic tractional retinal detachment in Chicago's county health system. PLoS One 14: e0220726, 2019.

15. Wang D, Bloomberg JD, Sobrin L, Goldstein D and Skondra D: Atypical herpes simplex virus type 2 acute retinal necrosis presentation with large subretinal lesion. Am J Ophthalmol Case Rep 15: 100501, 2019.

16. Quan SC and Skondra D: Case report: Varicella-zoster encephalitis with acute retinal necrosis and oculomotor nerve palsy. Optom Vis Sci 96: 367-371, 2019.

17. Sokol JT, Ferenchak K, Rosen DT, Schechet SA and Skondra D: Macular hole formation after pars plana vitrectomy for diabetic tractional retinal detachment. Ophthalmic Surg Lasers Imaging Retina 49: e256-e262, 2018

18. Baim AD, Movahedan A, Faroog AV and Skondra D: The microbiome and ophthalmic disease. Exp Biol Med (Maywood) 244: 419-429, 2019.

19. Schechet S, Hariprasad SM, Movahedan A and Skondra D: Use of Optical coherence tomography angiography in masqueraders of wet age-related macular degeneration and choroidal neovascularization. Ophthalmic Surg Lasers Imaging Retina 49: 80-85, 2018. 
20. Puzanov I, Diab A, Abdallah K, Bingham CO III, Brogdon C, Dadu R, Hamad L, Kim S, Lacouture ME, LeBoeuf NR, et al; Society for Immunotherapy of Cancer Toxicity Management Working Group: Managing toxicities associated with immune checkpoint inhibitors: Consensus recommendations from the Society for Immunotherapy of Cancer (SITC) Toxicity Management Working Group. J Immunother Cancer 5: 95, 2017.

21. Skondra D, Westerfeld C and Vavvas DG: Modified controlled encircling scleral buckle for retinal detachment. J Vitreoretin Dis 1: 314-316, 2017.

22. Ma J, Desai R, Nesper P, Gill M, Fawzi A and Skondra D: Optical coherence tomographic angiography imaging in age-related macular degeneration. Ophthalmol Eye Dis 9: 1179172116686075, 2017.

23. Skondra D, Nesper PL and Fawzi AA: Multimodal imaging of acute exudative polymorphous vitelliform maculpathy with optimal coherence tomography angiography and adaptive optics scanning laser ophthalmoscopy. Retin Cases Brief Rep 13: 195-198, 2019.

24. Simonett JM, Chan EW, Chou J, Skondra D, Colon D, Chee CK, Lingam G and Fawzi AA: Quantitative analysis of en face spectral-domain optical coherence tomography imaging in polypoidal choroidal vasculopathy. Ophthalmic Surg Lasers Imaging Retina 48: 126-133, 2017.

25. Roberts PK, Nesper PL, Onishi AC, Skondra D, Jampol LM and Fawzi AA: Characterizing photoreceptor changes in acute posterior multifocal placoid pigment epitheliopathy using adaptive optics. Retina 38: 39-48, 2018.

26. Heiferman MJ, Rahmani S, Jampol LM, Nesper PL, Skondra D, Kim LA and Fawzi AA: Acute posterior multifocal placoid pigment epitheliopathy on optical coherence tomography angiography. Retina 37: 2084-2094, 2017.

27. Dawood S and Skondra D: Monocular floaters and flashes. Dis Mon 63: 80-87, 2017.

28. Finn AP, Eliott D, Kim LA, Husain D, Wu DM, Young LH, Kim IK, Andreoli C, Skondra D, Vavvas DG, et al: Characteristics and outcomes of simultaneous bilateral rhegmatogenous retinal detachments. Ophthalmic Surg Lasers Imaging Retina 47: 840-845, 2016

29. Shah RS, Soetikno BT, Yi J, Liu W, Skondra D, Zhang HF and Fawzi AA: Visible-light optical coherence tomography angiography for monitoring laser-induced choroidal neovascularization in mice. Invest Ophthalmol Vis Sci 57: 86-95, 2016.

30. Callahan AB, Skondra D, Krzystolik M, Yonekawa Y and Eliott D: Wyburn-Mason syndrome associated with cutaneous reactive angiomatosis and central retinal vein occlusion. Ophthalmic Surg Lasers Imaging Retina 46: 760-762, 2015.

31. Asano MK, Papakostas TD, Palma CV and Skondra D: Visual outcomes of surgery for stage 4 and 5 retinopathy of prematurity. Int Ophthalmol Clin 54: 225-237, 2014.

32. Papakostas TD, Kohanim S, Skondra D, Lo K and Chodosh J: Medical management of Alternaria keratitis with endophthalmitis in a patient with a corneal graft. Clin Exp Ophthalmol 42: 496-497, 2014
33. Papakostas TD, Yonekawa Y, Skondra D and Vavvas DG: Traumatic chorioretinal rupture (sclopetaria). Int Ophthalmol Clin 53: 119-125, 2013.

34. Skondra D, Chang GC, Farber HW and Eliott D: Ophthalmologic diagnosis of exacerbation of idiopathic pulmonary arterial hypertension. Arch Ophthalmol 130: 1619-1621, 2012.

35. Hunter RS, Skondra D, Papaliodis G and Sobrin L: Role of OCT in the diagnosis and management of macular edema from uveitis. Semin Ophthalmol 27: 236-241, 2012.

36. Skondra D, Papakostas T and Vavvas DG: Enhanced depth imaging optical coherence tomography in age-related macular degeneration. Semin Ophthalmol 27: 209-212, 2012.

37. Skondra D, Papakostas TD, Hunter R and Vavvas DG: Near infrared autofluorescence imaging of retinal diseases. Semin Ophthalmol 27: 202-208, 2012

38. Chan RV, Yonekawa Y, Lane AM, Skondra D, Munzenrider JE, Collier JM, Gragoudas ES and Kim IK: Proton beam irradiation using a light-field technique for the treatment of choroidal hemangiomas. Ophthalmologica 224: 209-216, 2010.

39. Pallikaris A, Skondra D, Karavvela $M$ and Tsilimbaris $M$ : Confocal scanning laser tomography analysis of choroidal neovascularization and correlation with quantitative fluorescein angiography. Curr Eye Res 34: 319-327, 2009.

40. Skondra D, Noda K, Almulki L, Tayyari F, Frimmel S, Nakazawa T, Kim IK, Zandi S, Thomas KL, Miller JW, et al: Characterization of azurocidin as a permeability factor in the retina: Involvement in VEGF-induced and early diabetic blood-retinal barrier breakdown. Invest Ophthalmol Vis Sci 49: 726-731, 2008

41. Nakazawa T, Matsubara A, Noda K, Hisatomi T, She H, Skondra D, Miyahara S, Sobrin L, Thomas KL, Chen DF, et al: Characterization of cytokine responses to retinal detachment in rats. Mol Vis 12: 867-878, 2006.

42. Tsilimbaris MK, Charisis SK, Naoumidi T, Panteleontidis V, Skondra D, Christodoulakis E and Naoumidi I: Contact transscleral ciliary body photodynamic therapy in pigmented rabbits using verteporfin and diode laser: Evaluation of treatment parameters. Curr Eye Res 31: 577-585, 2006.

43. Pallikaris A, Skondra D and Tsilimbaris M: Intraobserver repeatability of macula measurements by confocal scanning laser tomography. Am J Ophthalmol 139: 624-630, 2005.

44. Skondra D: Early gut microbiome profile in high-risk preterm infants with and without retinopathy of prematurity. In: Proceedings of The Retina Society 2019 Annual Meeting, London, 2019.

This work is licensed under a Creative Commons Attribution-NonCommercial-NoDerivatives 4.0 International (CC BY-NC-ND 4.0) License. 